
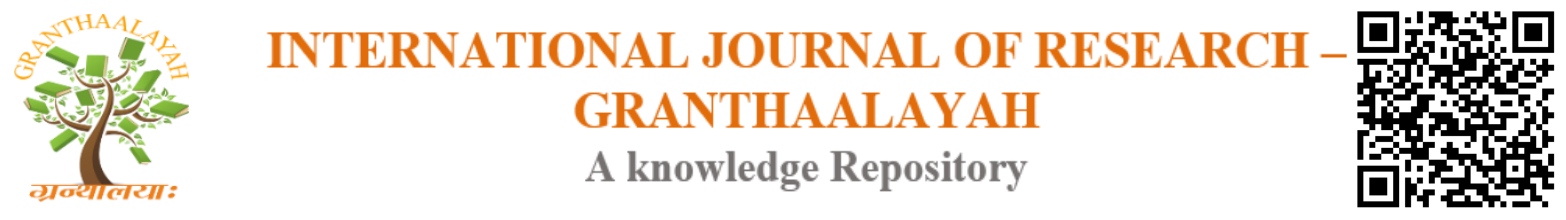

Science

\title{
BIOMAGNETISM AS FACTOR IN RED BLOOD CELLS DEFORMATION
}

\author{
Abraham A. Embi Bs*1 \\ *11 13442 Sw 102 Lane Miami, Florida USA 33186, Citizen Scientist
}

\begin{abstract}
The purpose of this manuscript is to report in vitro experiments showing the role of pulsed biomagnetic fields tissues cross-talk between Red Blood Cells (RBCs) and human hairs. Both tissues have been reported to express magnetic properties, ie: RBCs diamagnetic and paramagnetic forces and the hair follicle pulsed diamagnetic forces. This biomagnetic cross-talk is reported as a novel factor in RBCs deformation. In the in vitro experimental model herein used, other forces such as keratin biomagnetism, hydrophilic and hydrophobic properties of the hair shaft may also play a role in the deformation. Presently teardrop red blood cells found in blood smears; and oriented in the same direction are attributed to mechanical artifacts introduced during slide preparations. The data presented in this manuscript supports the new principle of biomagnetic cross talk forces as factor in replicating RBCs deformities.as described in Optical Tweezers Trapping.
\end{abstract}

Keywords: Dacrocytes; Teardrop Cell; Tissue Ccross-talk; RBCs Deformation; Diamagnetism; Paramagnetism; Pulsed Biomagnetism; Optical Trap Tweezers.

\section{Glossary}

Pulsed Biomagnetic Cross-Talk= Reciprocal forces (attraction/repulsing) forces between tissues. Example is hair and blood causing red blood cells deformation.

Dacrocyte $=$ Defined as red blood cells in the shape of teardrop.

Diamagnetism= Material repulsion to an external magnetic field.

Optical TrapTweezers= "Very sensitive tool, based on photon momentum transfer. Used for cell trapping and manipulation. (6)

Paramagnetism= Material attracted to external magnetic field. ie: iron to magnet.

Replicating= To make something identical again, ie: Deformed red blood cells.

Cite This Article: Abraham A. Embi Bs. (2018). "BIOMAGNETISM AS FACTOR IN RED BLOOD CELLS DEFORMATION.” International Journal of Research - Granthaalayah, 6(12), 46-57. https://doi.org/10.29121/granthaalayah.v6.i12.2018.1245. 


\section{Introduction}

The present paradigm of tissue cross-talk is defined as the molecular communication between body tissues or parts (1). Biomagnetism as a tissue cross-talk theory was implied by D Cohen et al in 1975 by stating "Measurements of the field around the human body can yield new information about the organs which generate current, not available to surface electrodes" (2), this concept has not fully yet reached "mainstream" medical journals. To date few papers have addressed biomagnetism as a factor in tissue cross-talk, one exception is the discovery of a mechanism explaining Low Level Transcutaneous Electrical Stimulation of the Vagus Nerve reversing fibrosis buildup in the cardiac myocytes (3). The main purpose of this manuscript is to introduce a new finding, which is pulsed biomagnetic fields as factor contributing to the genesis of Dacrocytes; that are deformed red blood cells (RBCs) in the shape of teardrops and observed microscopically in patients blood smears (4). These odd shaped cells are often associated with an abnormal spleen; bone marrow infiltration or hematologic and non-hematologic malignancies. Teardrop cells with tails pointing in the same direction are reported as artifacts (5); that are said to be as result of mechanical distortion during the smear preparation. This manuscript introduces $n=4$ experiments on different individuals ( 2 males, ages 44 and 76; and 2 females ages 42 and 72). All participants developed Dacrocytes (in the in vitro experiments) triggered by pulsed biomagnetical cross-talk between two body tissues, namely the hair shaft and blood. The images herein reported correlate with images of RBCs deformation induced by the laser based "Optical Tweezers" method (6).

\section{Material and Methods}

Three materials were used as ancillary testing to evaluate the impact of the biomagnetic cross-talk between blood and materials (salt crystals, ground pepper and human skin keratin flakes). Two endogenous materials, such as human hairs and fresh blood drops were also used. Recording equipment used for data storage and further evaluation was a video microscope (Celestron LCD Digital Microscope II model \#44341 and images downloaded into an Apple System Photo Application.

\subsection{First Approach}

\subsubsection{Blood Smear Preparation}

A finger stick allowed for the milking of two drops of blood, then placed on a clean $25 \times 75 \times 1 \mathrm{~mm}$ glass slide. The mechanical smear was done as per published instructions from the USA center for disease control.

\subsubsection{Standard Blood Smear}

It is emphasized that as soon as the smear is done, moisture will evaporate from the thin smear; therefore the particles tested will fail to interface with the RBCs in dry smears. In all experiments herein presented, the materials tested were gently placed on the slide within 10 seconds of smearing. 


\subsection{Ancillary Testings}

\subsubsection{Symmetrical Blood Repulsion (Salt Crystal)}

Non-tissue material particles such as salt crystals and ground pepper flakes were placed on each individual wet smear $(n=4)$ and then viewed in the video-microscope. (Figs 1,2,3 \& 4)).

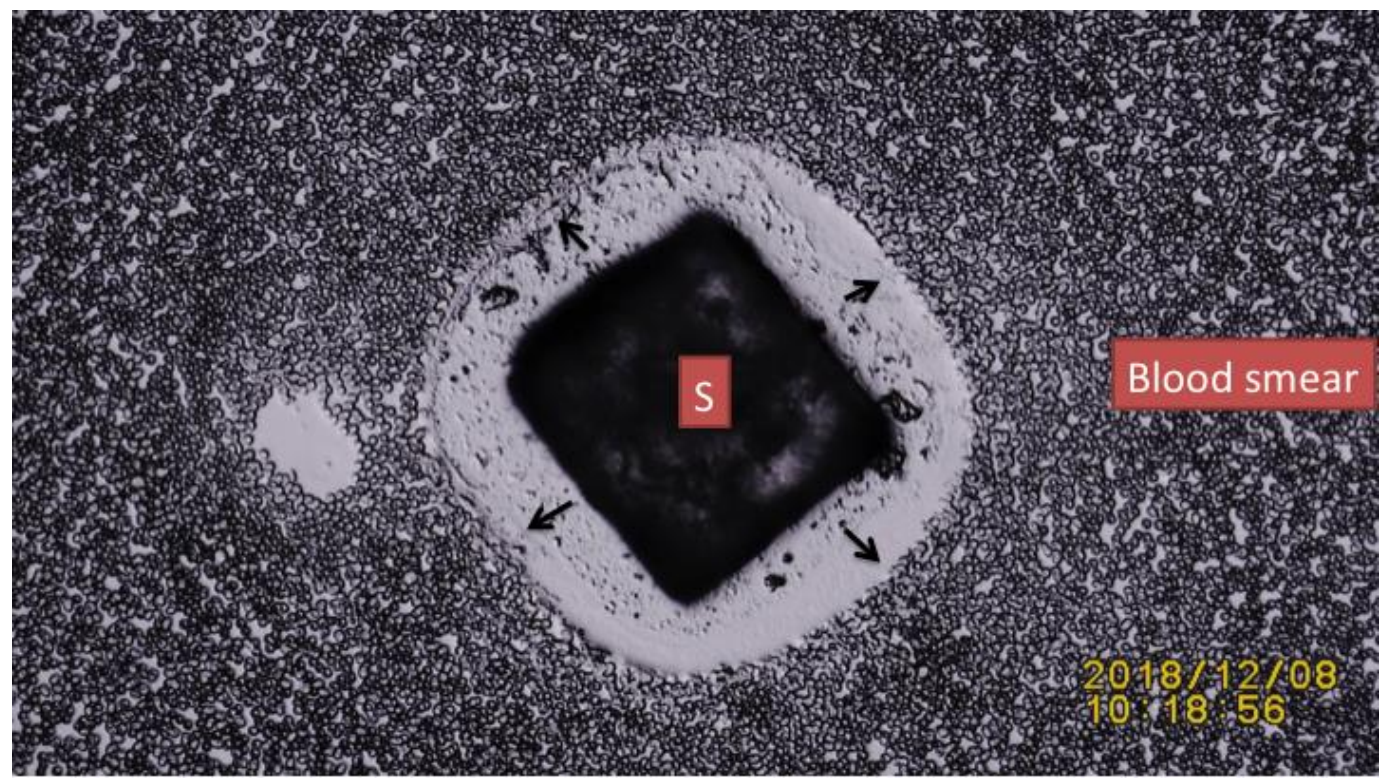

Figure 1: Salt crystal placed on wet blood smear slide. $S=$ Salt crystal. Black arrows= Symmetrical blood displacement, giving the appearance of a circular shape. This image obtained immediately (approximately 10 seconds) after gently placing a salt crystal on a wet blood smear.

\subsubsection{Symmetrical Blood repulsion (Liquid Butter)}

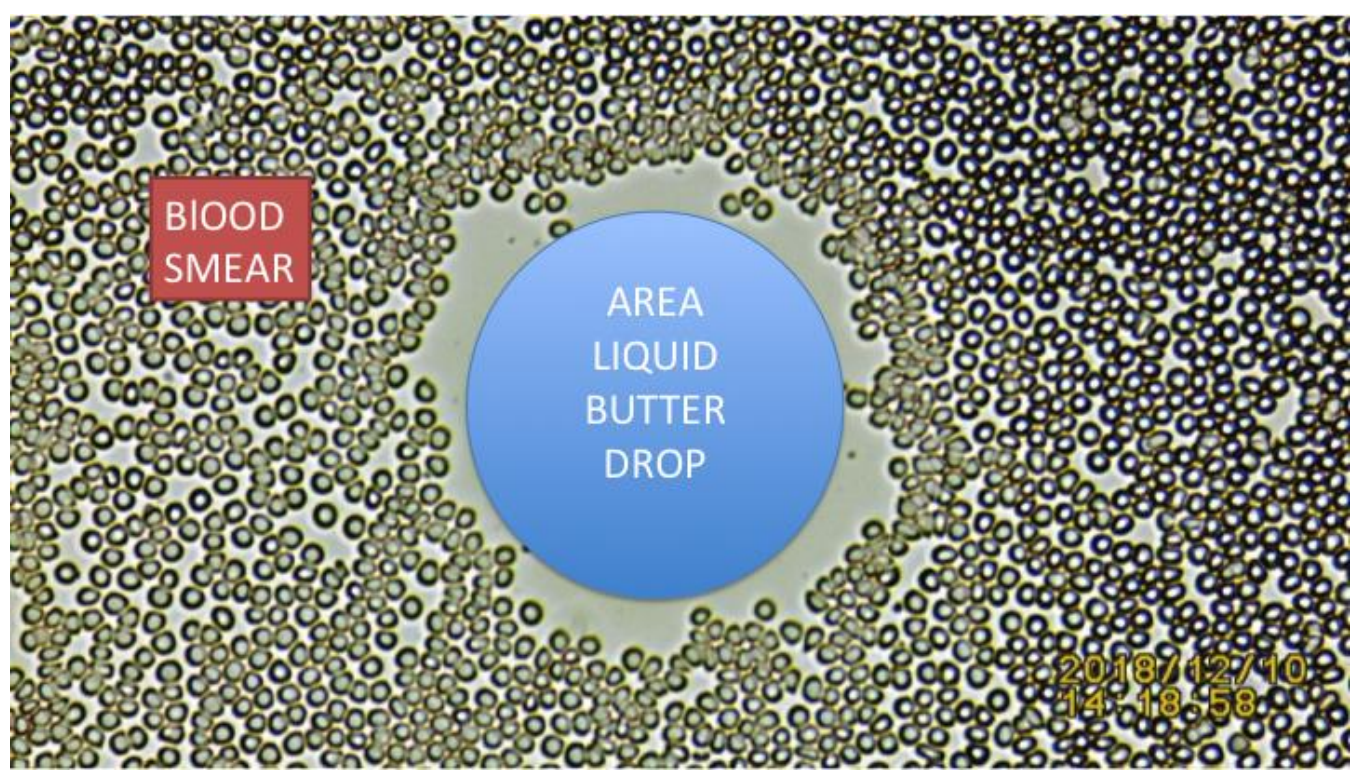

Figure 2: Liquid butter drop on smear. Added blue circle mimicking liquid butter drop area. Notice symmetry of RBCs displacement. 


\subsubsection{Asymmetrical Blood Repulsion (Ground Pepper Flakes)}

In this smear ground pepper flakes were gently placed on a wet blood smear. There was an asymmetrical repulsion of the thin blood smear by this material.

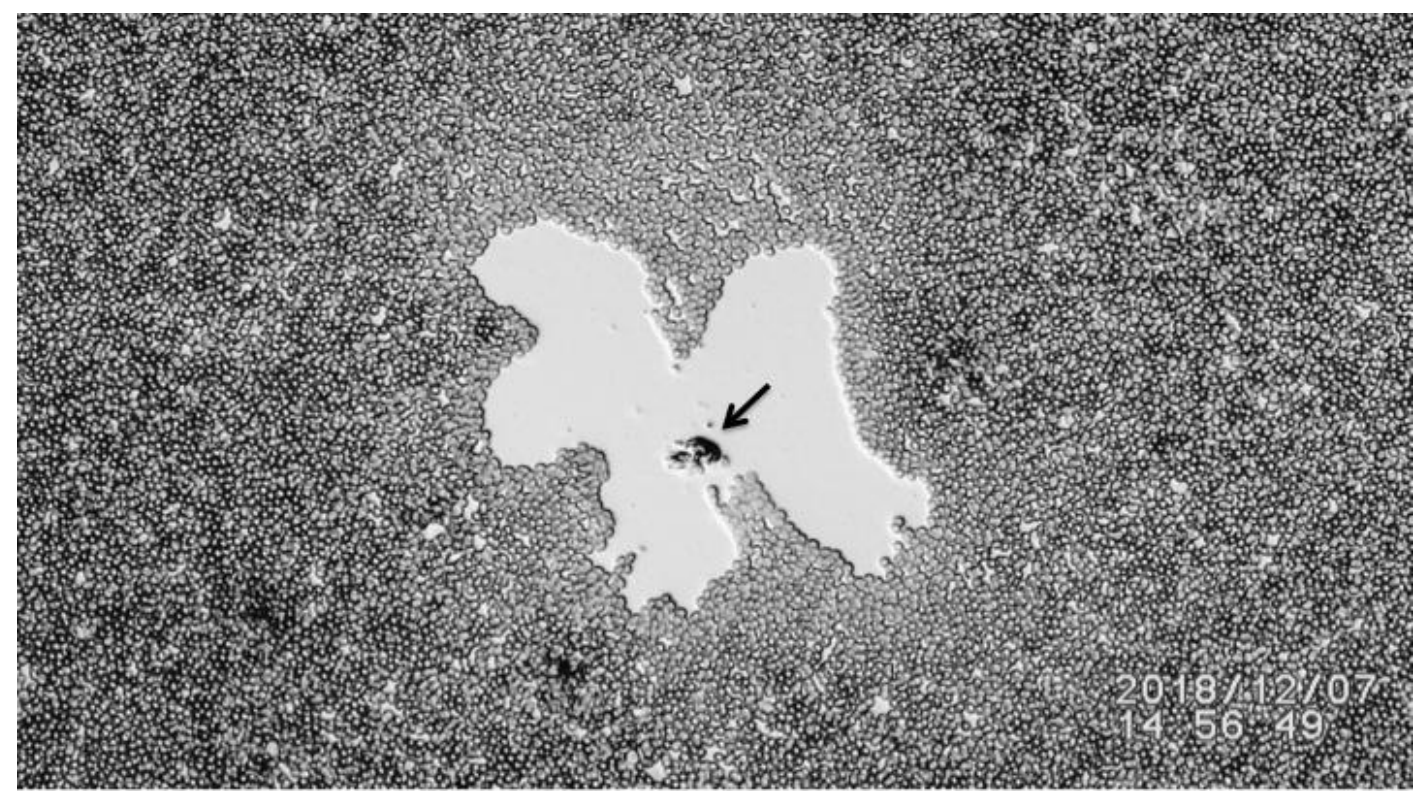

Figure 3: Showing author's RBCs in smear asymmetrically displaced by the minute ground paper flake particles. Example of biomagnetic repulsion of blood by an exogenous material.

\subsubsection{Asymmetrical Blood Repulsion by Keratin}

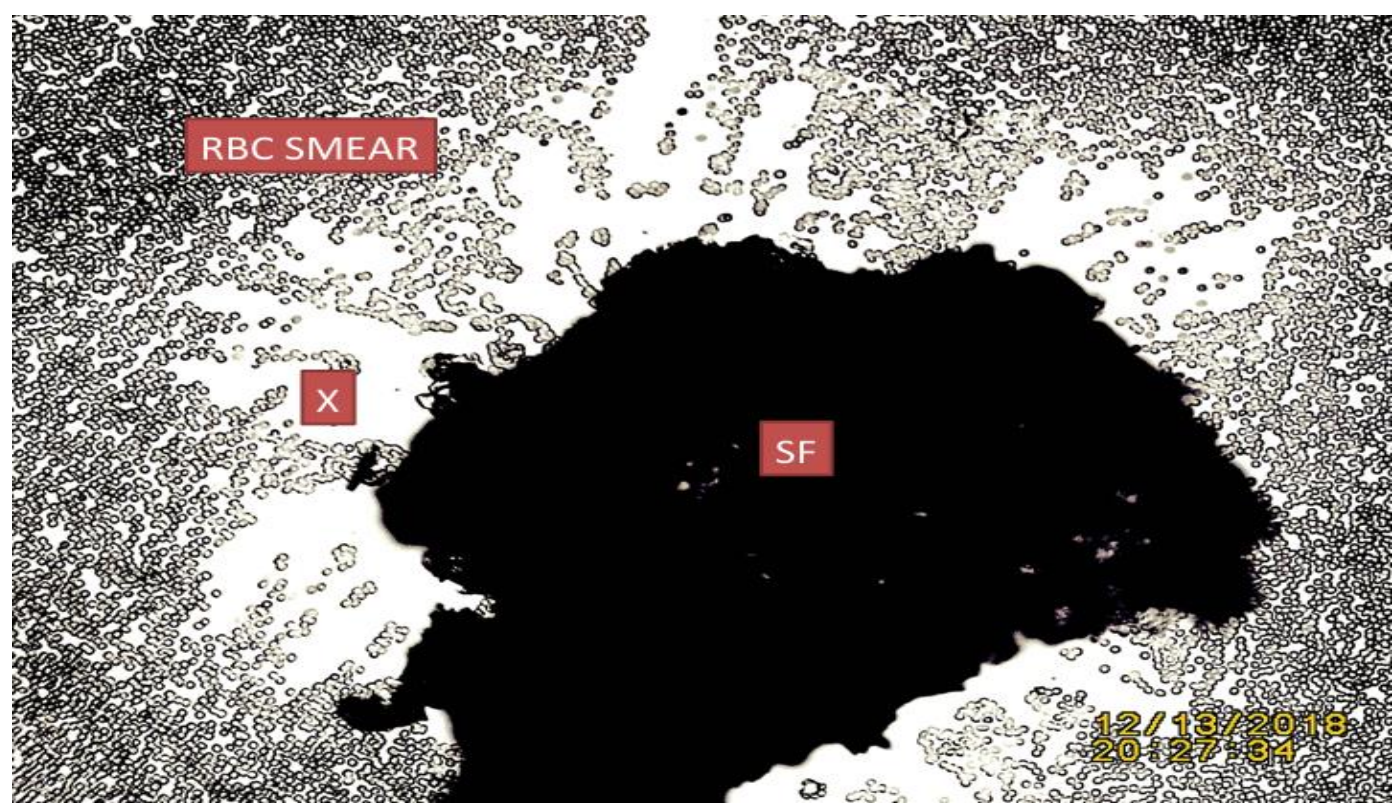

Figure 4: Example of Keratin repelling RBCs. SF= Dry white skin flake consisting of keratin, placed on blood smear. $\mathrm{X}=$ Notice asymmetry of RBCs displacement circumventing flake. 


\subsubsection{Figure Below Human Hair and Blood Cross-Talk Experiments}

\section{Hair Follicle Causing Asymmetrical Blood Repulsion}

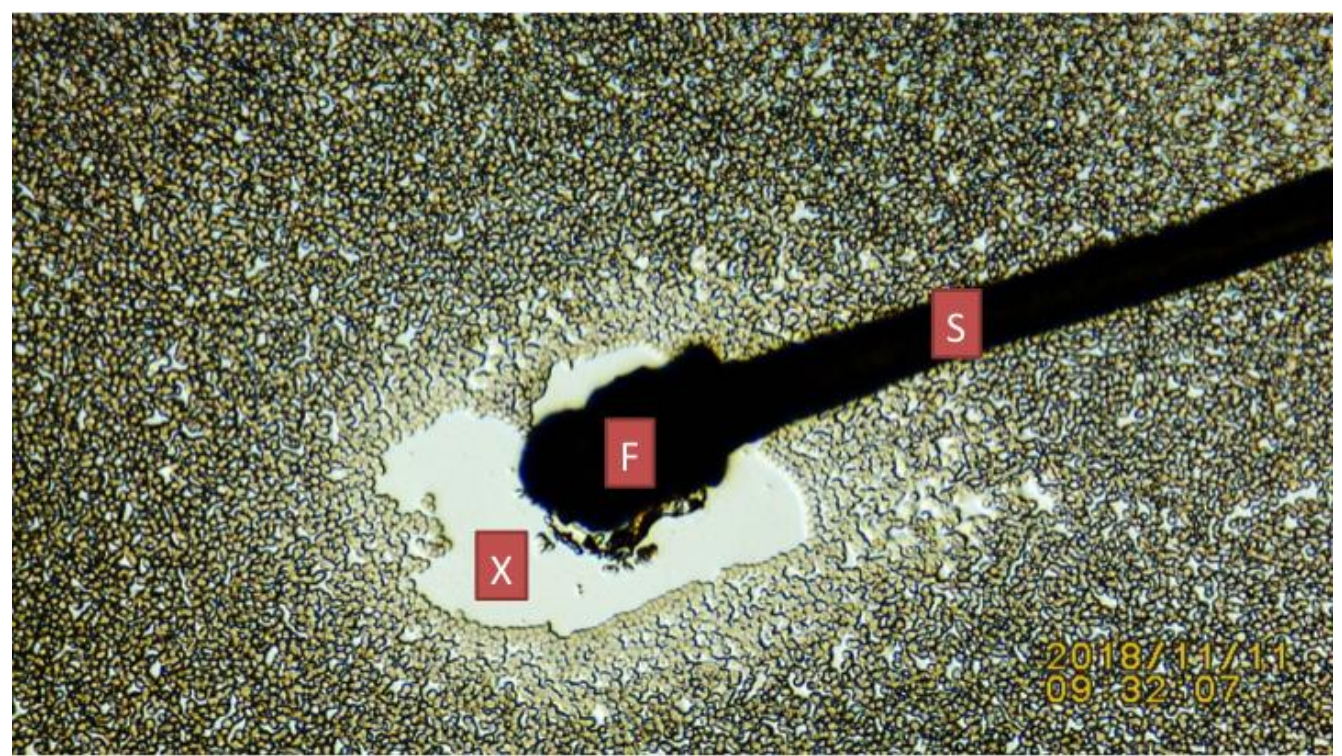

Figure 5: Showing image of hair placed on wet blood smear. Notice the asymmetrical blood repulsion pattern. $\mathrm{F}=$ Follicle $\mathrm{X}=$ Biomagnetic Zone $\mathrm{S}=$ Hair shaft (not in contact with blood smear). Notice the asymmetrical displacement of the RBCs, intrinsic hair follicle Pulsed Biomagnetism.

\subsection{Second Approach}

\subsubsection{Hair Shaft Experiments}

\section{Blood Drops on Forearm Covered by Glass Slide (Passive Approach)}

This approach could be labeled a non-motion (passive) non-spreading technique, in other words, opposite to the standard smear technique where the blood drops are mechanically spread by a continuous motion; in this technique the blood drops are "passively" placed on the skin, then covered by a $25 \times 75 \times 1 \mathrm{~mm}$ slide that is tape secured on the forearm and vertically removed 20 minutes later. 


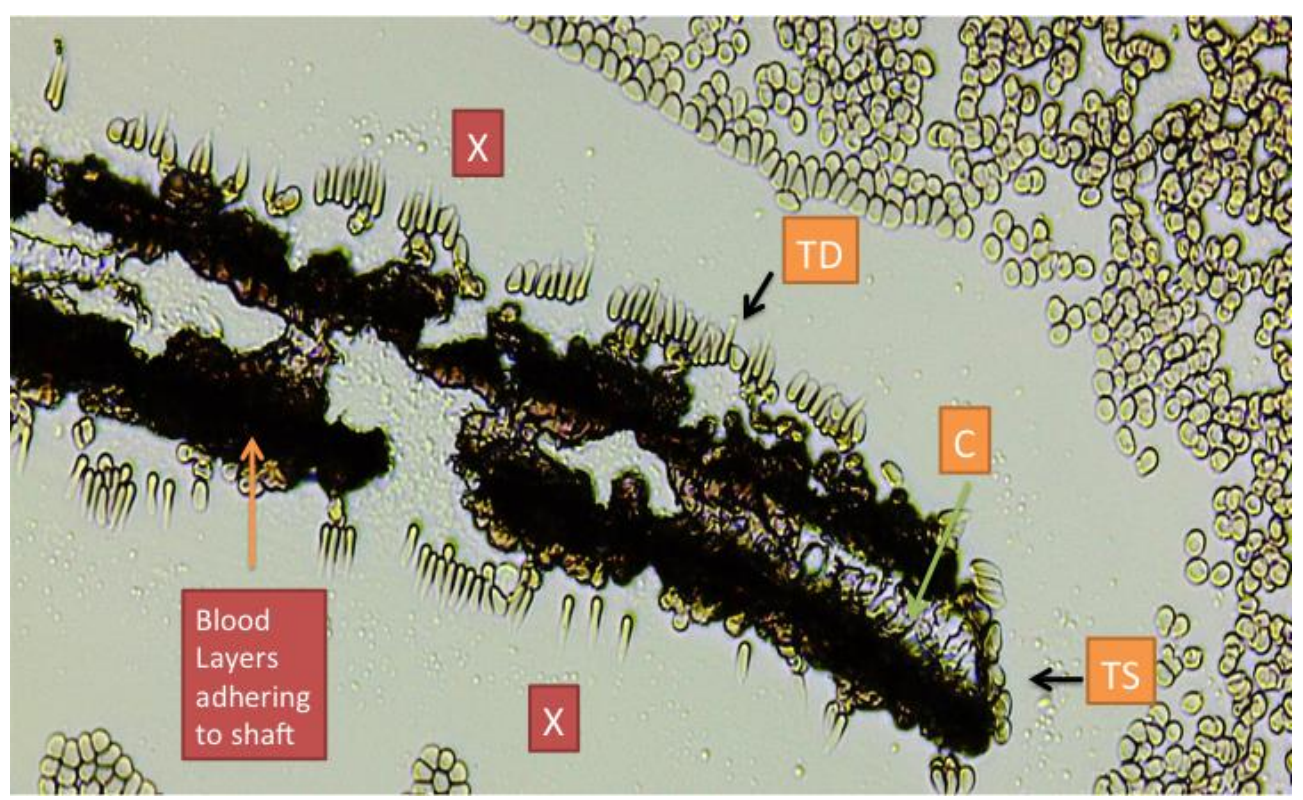

Figure 6: Male subject. Hair shaft outline adhering to slide, after shaft proper removed from smear. $\mathrm{C}=$ Cuticles adhered by blood $\mathrm{TD}=$ Aligned teardrop shape $\mathrm{RBCs} .=$ Dark area surrounding hair shaft from clumping blood adhering to hair shaft. TS= Single layer of RBCs adhering to cut shaft end. $\mathrm{X}=$ Pulsed biomagnetic Field emitted by tissue cross-talk.

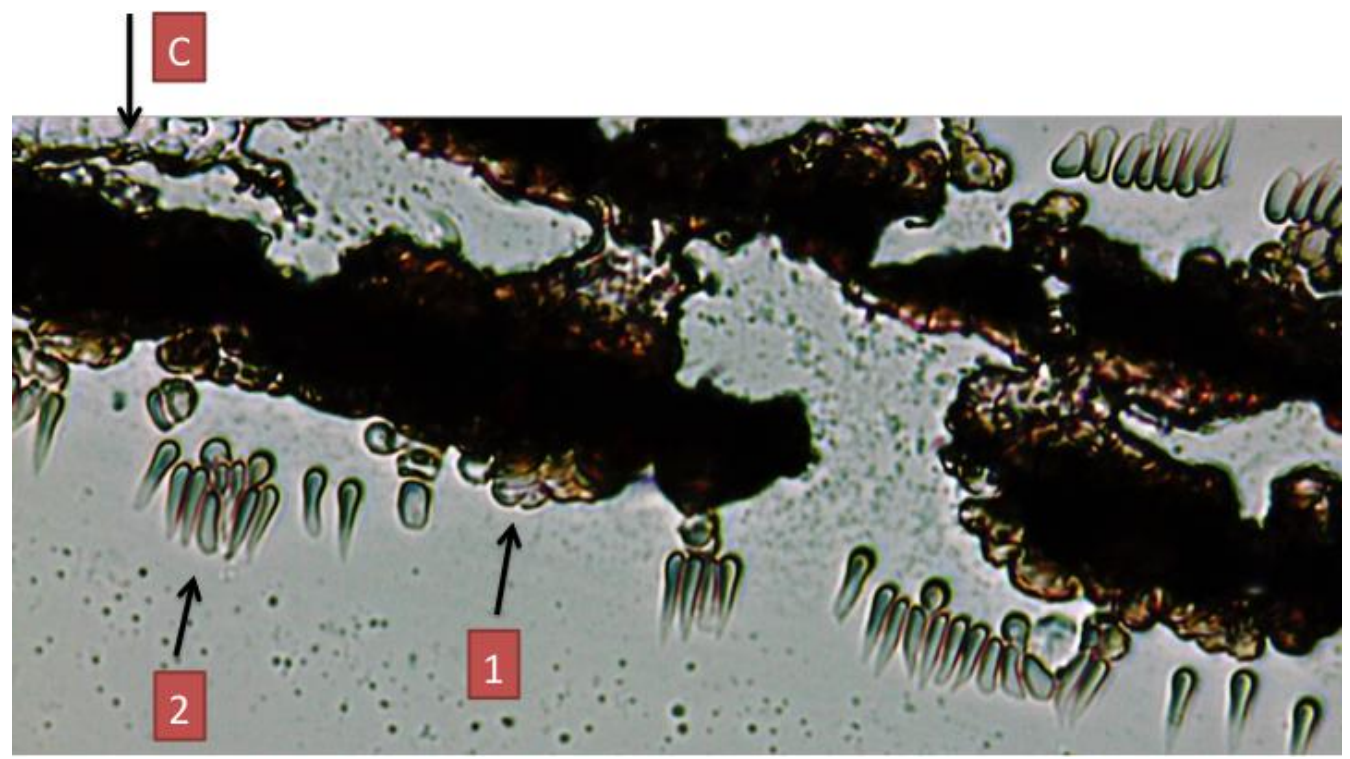

Figure 7: Proposed sequence of events. Hair shaft outline adhering to slide, after removal from smear.showing: 1= Normal RBCs clumping (attracted by shaft) 2= RBCs deformed in teardrop shape are also attracted and deformed as teardrops. $\mathrm{C}=$ Hair cuticles.

\section{Results}

A tabletop microscopy method is introduced showing pulsed biomagnetic cross-talk of two human tissues, namely hair and blood causing cells deformities. Molecular tissue cross-talk has been previously identified and the incident of biochemical exchanges rank in the millions (7). Biomagnetical tissue cross-talk has also recently been described in the literature to a lesser degree. 
Example is the inhibition by the hair shaft biomagnetism on fibrin formation in blood (8). In the present manuscript biomagnetism cross-talk induces molecular changes in one of the tissues, in this case the heterogeneous distortions in RBC shapes. These changes seem to involve the RBC membrane. The mechanism behind these results is discussed below.

\section{Discussion}

The hair shaft is composed of $95 \%$ keratin, which has been found to have an affinity to the protein enzyme catalase (present in RBCs) (9). The two tissue tested have similar magnetic properties, for example, both keratin and hemoglobin are diamagnetic as well as paramagnetic as described $(10,11,12)$. The hair shaft also being hydrophobic (repelling water), as well as hydrophilic (attracting water) (13). Ground pepper fragments also attracted and deformed RBCs in the smear. Pepper fragments lack keratin; instead they have paramagnetic properties.

\subsection{Novel Factor Inducing Cells Deformation}

This manuscript introduces a novel factor in replicating deformation of RBCs as teardrop shapes and others. These changes were found in in vitro experiments on $n=4$ individuals. Currently, when a blood test is done and teardrop cells are seen aligned in the same direction, is reported by pathologist as artifacts. This is attributed to a mechanical forces applied when preparing a smear. The present manuscript exhibits via numerous examples, that teardrop RBCs aligned in the same direction were consistently triggered by non-mechanical forces, such as paramagnetisc and possibly diamagnetisc forces; or also possibly hydrophobic and hydrophilic properties in the materials used for this demonstration. The hair shaft consists of 95\% keratin that has been shown to bind with the protein enzyme catalase ever present in RBCs. Ancillary testing (Figure 4) shows the asymmetrical complex repulsion of RBCs by keratin. Ground pepper particles also asymmetrically repel RBCs (Figures 3 and S3 and S4), this is attributed to the paramagnetic property of ground pepper "having three paramagnetic species that are located mostly at the seed surface" (14). Both keratin and human RBCs are also known to have paramagnetic and diamagnetic properties. As an additional note, in the small cohort $n=4$ the two males displayed multiple provoked aligned teardrop cells (50+ bilaterally); whereas the two females average 4 teardrops per field. (Please revisit figure 4 in text and last supplementary images posted after references). There is precedent for sex differences in hemolysis of RBCs as previously published: "Based on our findings, we propose that the sex effect is intrinsic to the erythrocyte, involves membrane function, and is highly conserved in mammalian evolution (15).

\subsection{Summarizing by Process of Elimination}

Using glass slides and microscopy, two tabletop methods are described causing deformation of RBCs. The variety of material used and data presented points at biomagnetic forces present in all three materials as factor in the reshaping of RBCs. Of significance in reaching a conclusion is the attraction of the iron present in RBCs attracted by the paramagnetic ground pepper flakes. The latter eliminates diamagnetic forces present in keratin; although the hair follicle intrinsic pulsed biomagnetism also induces RBCs changes. Herein introduced is a novel tabletop method demonstrating that paramagnetic and diamagnetic forces coss-talk replicate the "Optical Blood Tweezers Trap" effect on RBCs deformation. 
The questions arise:

1) Are teardrop cells formation sex linked? After all it appears that the RBC membrane is involved in our study. Further research appears to be justified.

2) Are we seen a novel tabletop method biomagnetic cross-talk replicating the "Optical Tweezers Trap" induced RBCs deformation?

The data seem to confirm both questions.

\section{References}

[1] Lee, D. E., Kehlenbrink, S., Lee, H., Hawkins, M. \& Yudkin, J. S. (2009). Getting the message across: mechanisms of physiological cross talk by adipose tissue. Am J Physiol Endocrinol Metab 296, E1210-1229.

[2] D. Cohen et al. (1975) Measurements of the magnetic fields produced by the human heart, brain and lungs. IEEE Transactions on Magnetics, Volume: 11, Issue: 2, pp $694-700$.

[3] Embi AA. (2018). "HAIR AND BLOOD ENDOGENOUS LOW LEVEL BIOMAGNETIC FIELDS CROSS-TALK EFFECTS ON FIBRIN INHIBITION AND ROULEAU FORMATION." International Journal of Research - Granthaalayah, 6(11), 200-208. https://doi.org/10.5281/zenodo.1845985.

[4] Center for Disease Control. USA. Suggested technique for Blood Smear. https://www.cdc.gov/dpdx/resources/pdf/benchAids/malaria/Malaria_procedures_benchaid.pdf.

[5] The American Association of Hematologists. Image Bank \# 00060311. 11/29/15

[6] Killian JL, Ye F, Wang MD. (2018) Optical Tweezers: A Force to Be Reckoned With. Cell. 2018 Nov 29;175(6):1445-1448. PMID: 30500527 doi: 10.1016/j.cell.2018.11.019.

[7] Aranda, Bruno et al. (2011) "PSICQUIC and PSISCORE: accessing and scoring molecular interactions", Nature methods vol. 8,7 528-9. 29 Jun. 2011, doi:10.1038/nmeth.1637

[8] Embi AA. (2018). "HAIR AND BLOOD ENDOGENOUS LOW LEVEL BIOMAGNETIC FIELDS CROSS-TALK EFFECTS ON FIBRIN INHIBITION AND ROULEAU FORMATION." International Journal of Research - Granthaalayah, 6(11), 200-208. https://doi.org/10.5281/zenodo.1845985.

[9] Embi AA. (2018). "TRANSFER OF HUMAN HAIR EXOCUTICLES ONTO GLASS BY LIQUID CATALASE IN CONTACT WITH WHOLE BLOOD (REPORTING BLOOD CLUMPING FORMATION WHEN CONTACT WITH KERATIN AS POSSIBLE FACTOR IN ATHEROSCLEROSIS).” International Journal of Research - Granthaalayah, 6(10), 187-193. https://doi.org/10.5281/zenodo.1476724.

[10] Langbein L, Schweizer J. Keratins of the human hair. (2005) follicle.Int Rev Cytol. 243:1-78. PMID:15797458 DOI:10.1016/S0074-7696(05)43001-6

[11] Pauling L, Coryell CD. (1936) The magnetic properties and structure of hemoglobin, oxyhemoglobin and carbon monoxyhemoglobin. Proc Natl Acad Sci 1936; 22:210-216. (first paper describing and explaining the diamagnetic and paramagnetic properties of oxy- and deoxyhemoglobin respectively)

[12] Bren, K. L., Eisenberg, R., \& Gray, H. B. (2015). Discovery of the magnetic behavior of hemoglobin: A beginning of bioinorganic chemistry. Proceedings of the National Academy of Sciences of the United States of America, 112(43), 13123-7.

[13] N Baghdadli, G S Luengo and L Recherche (2008) A closer look at the complex hydrophilic/hydrophobic interactions forces at the human hair surface. Journal of Physics: Conference Series, Volume 100, Part 5.

[14] Nakagawa, K., \& Epel, B. (2014). Locations of radical species in black pepper seeds investigated by CW EPR and 9GHz EPR imaging. Spectrochimica acta. Part A, Molecular and biomolecular spectroscopy, 131, 342-6. 
[15] Kanias, T., Sinchar, D., Osei-Hwedieh, D., Baust, J. J., Jordan, A., Zimring, J. C., Waterman, H. R., de Wolski, K. S., Acker, J. P., ... Gladwin, M. T. (2016). Testosterone-dependent sex differences in red blood cell hemolysis in storage, stress, and disease. Transfusion, 56(10), 25712583.

\section{Appendix}

\section{Supplementary Relevant Videos and Images}

\section{Examples of RBC Deformation by Biomagnetic Forces}

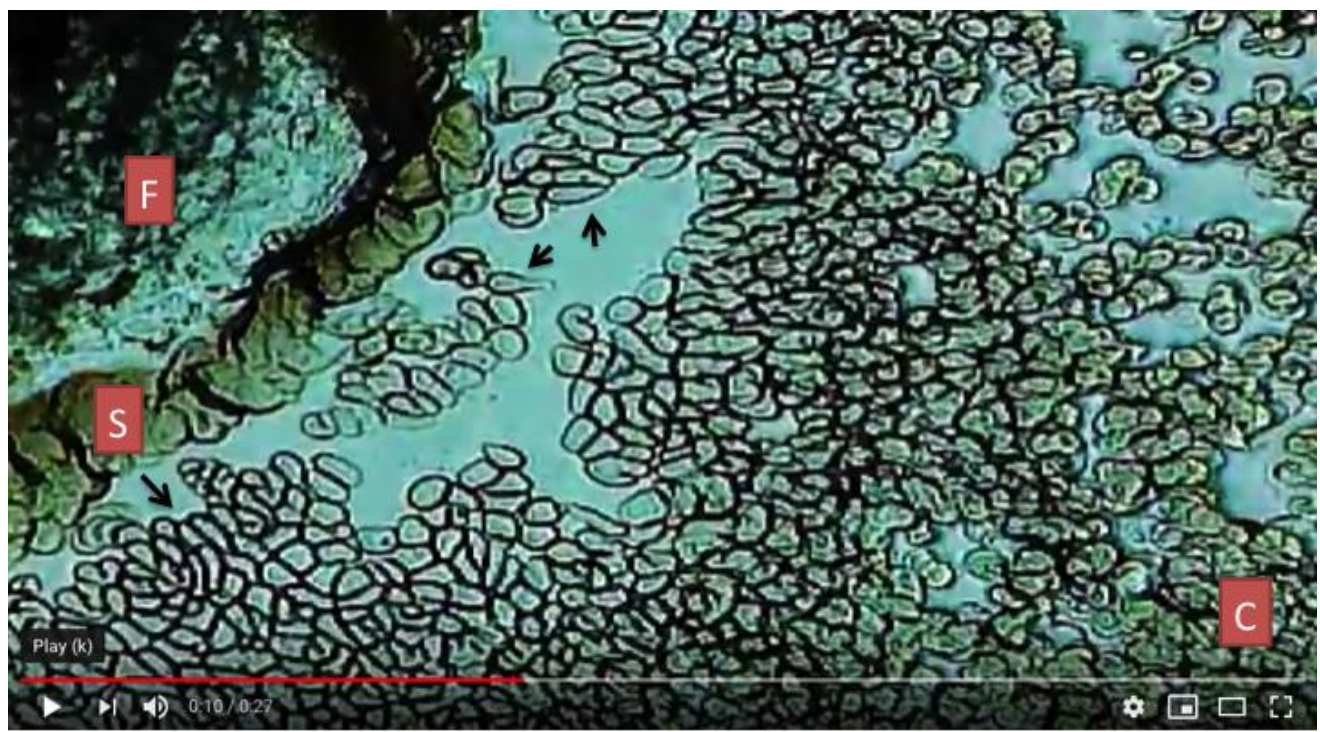

Figure S1: Tissue cross-talk. Microphotograph of still frame showing cellular heterogenous reshaping caused by the intrinsic pulsating electromagnetic field (PEMFs) of a human hair follicle placed on drops of human blood $\mathrm{F}=$ Distal Follicle. $\mathrm{S}=$ Sebum coat. Black Arrows= Pointing at reshaped RBCs. $\mathrm{C}=$ Coagulated blood seen in lower right corner away from EMFs range. Please visit video link:https://youtu.be/ErBiwoXgxRY or scan QR code below:

\section{Video Recording Demonstrating Pulsed Biomagnetism of Hair Folicle}

Please click on link or scan QR code below.

https://youtu.be/8pipWJVytrk 


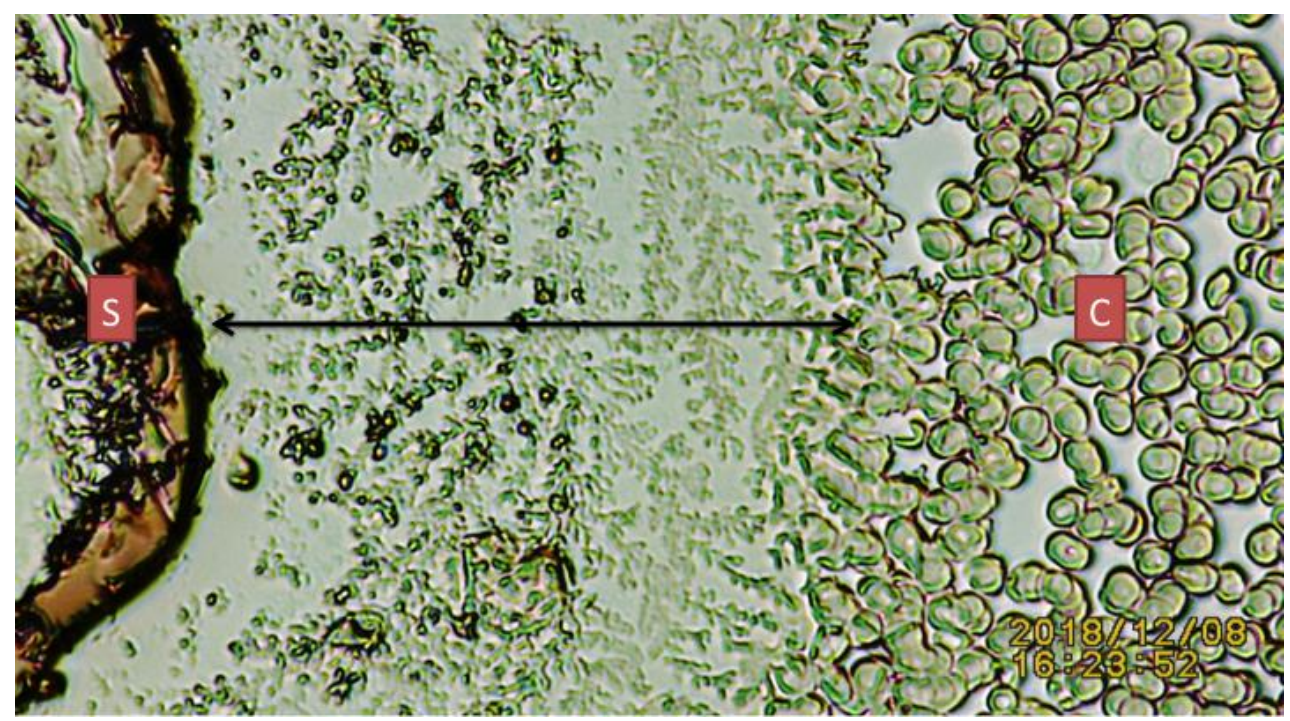

Figure S2: Amplified edge of removed salt crystal and absence effect on RBC deformation. Bidirectional black arrow identifying the symmetrical mechanical blood displacement by a biomagnetic inert material

\section{Asymmetrical Displacement of Blood Smear}

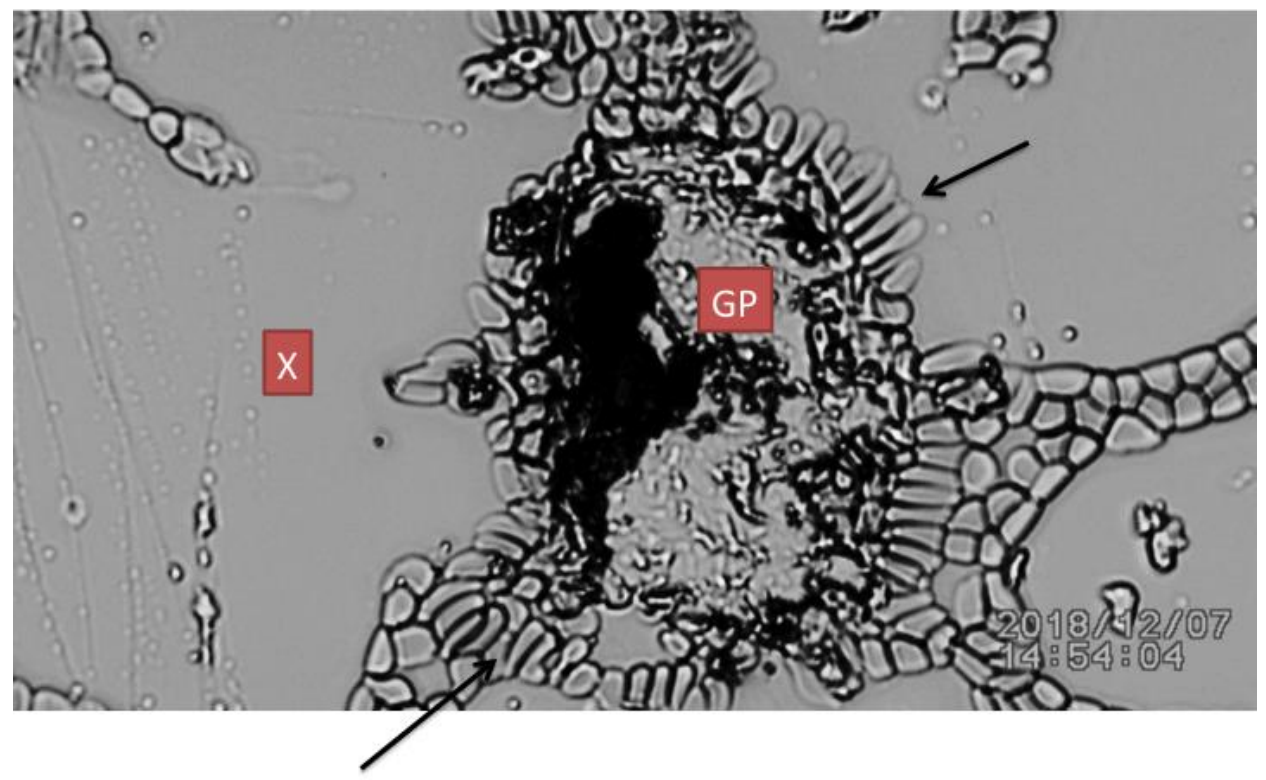

Figure S3: Ground pepper flake placed on wet blood smear- Notice asymmetrical dispersion of RBCs theorized to be as result of cross-talk Low Level Paramagnetic Magnetic Fields emitted by the flake, GP= Ground Pepper Flake. Black arrows= Pointing towards deformed RBCs attracted by the pepper intrinsic magnetism. $\mathrm{X}=$ Area void of RBCs. 


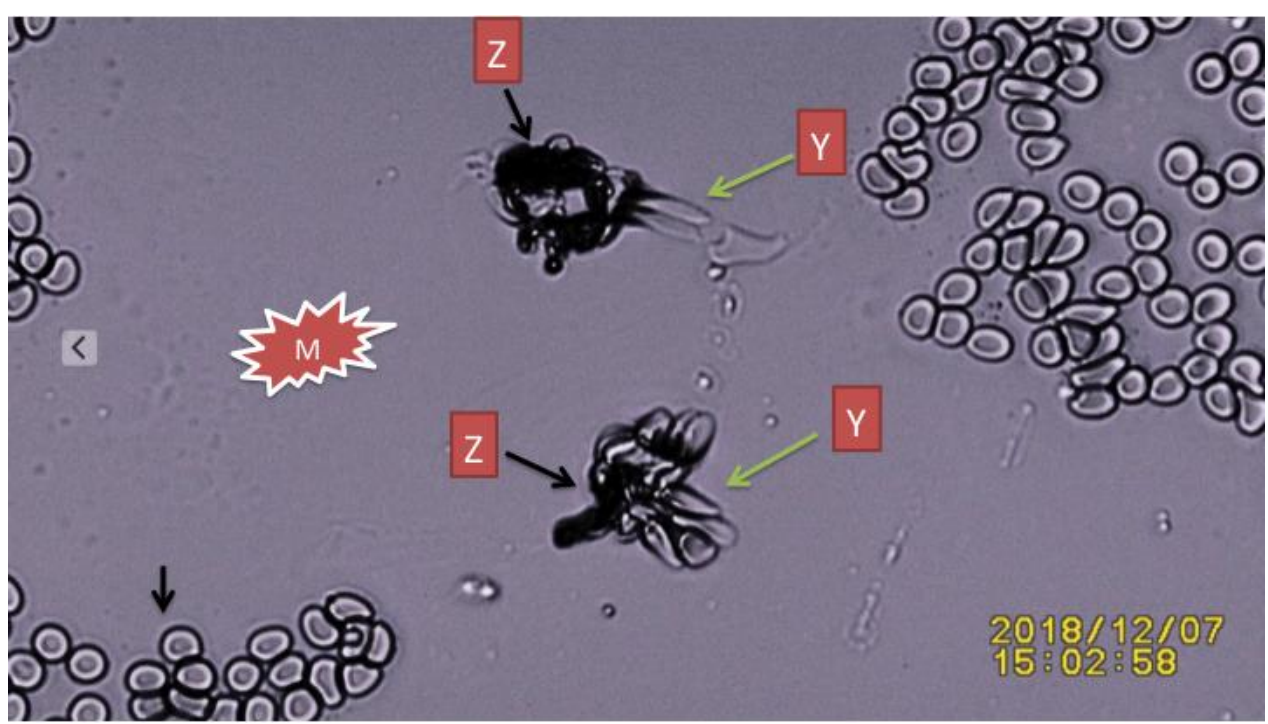

Figure S4: Amplified ground pepper flakes added to wet smear asymmetrically repelling blood; and inducing tear drop RBCs adhered to flake. Black Arrow= Repelled RBCs. Y= Teardrop shape RBCs adhering to flake. $Z=$ Ground pepper flake $M=$ Magnetic fields Pepper.

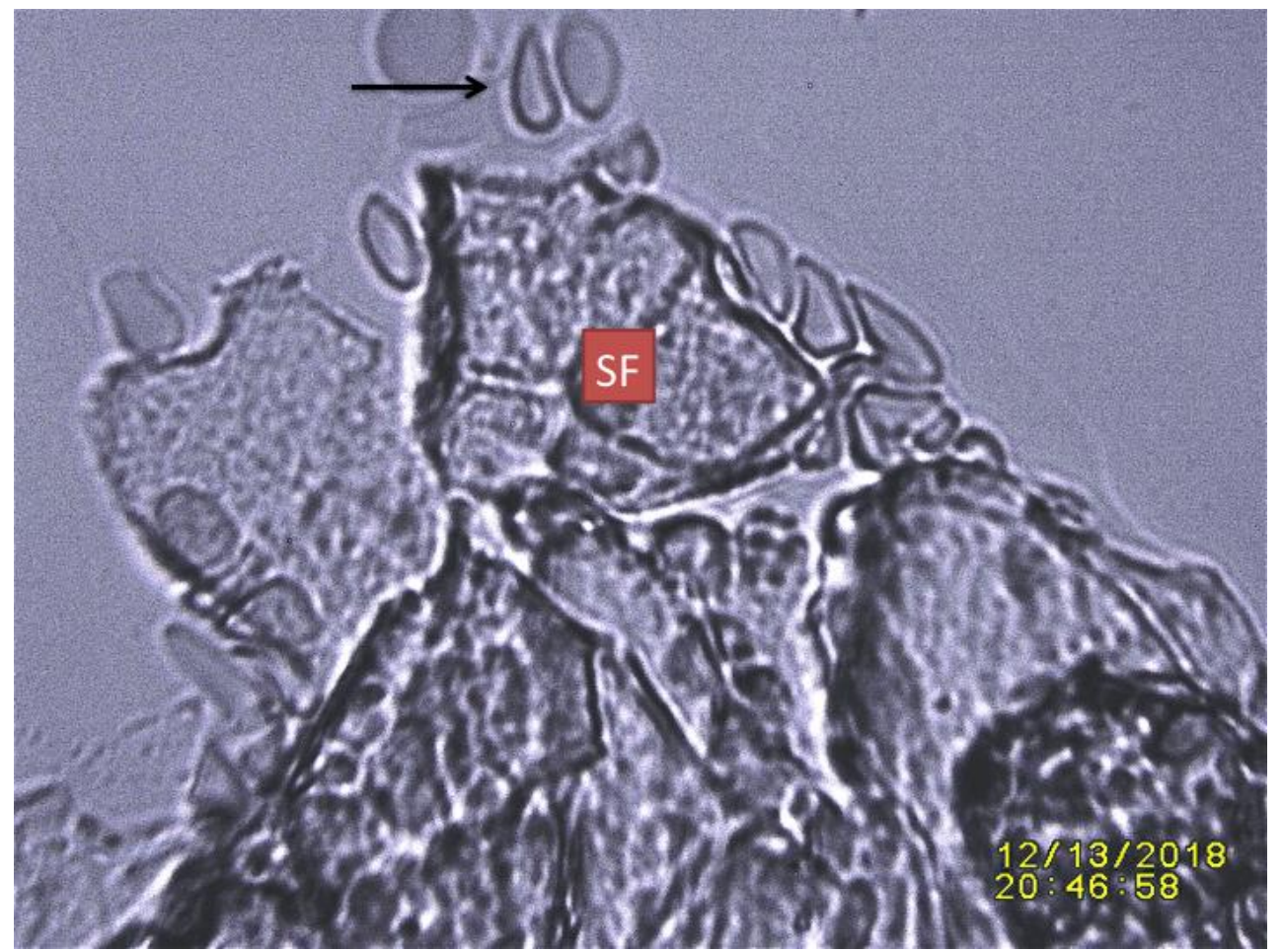

Figure S5: Amplified image of human skin keratin flake causing teardrop cells in blood smear. $\mathrm{SF}=$ Skin Fake. Black arrow= Teardrop cell. 


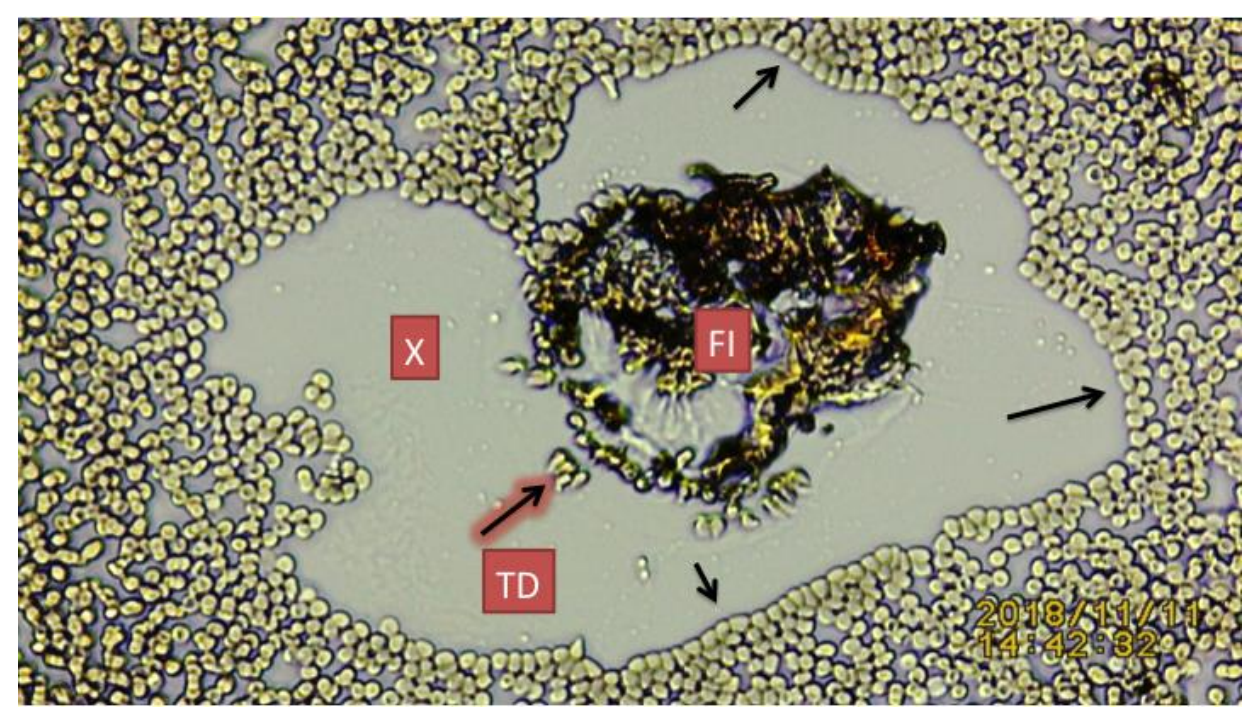

Figure S6: Image of removed hair seen in text Figure 5. Showing follicle imprint removed from glass slide.FI= Follicle imprint $\mathrm{X}=$ Diamagnetic Zone Black arrows= Asymmetrical displaced RBCs. $\mathrm{TD}=$ Teardrop cells attracted to follicle.

Image reproduced and re-labeled from: Abraham A. Embi Bs. (2018). "HAIR AND BLOOD ENDOGENOUS LOW LEVEL BIOMAGNETIC FIELDS CROSS-TALK EFFECTS ON FIBRIN INHIBITION AND ROULEAU FORMATION." International Journal of Research Granthaalayah, 6(11), 200-208. https://doi.org/10.5281/zenodo.1845985.

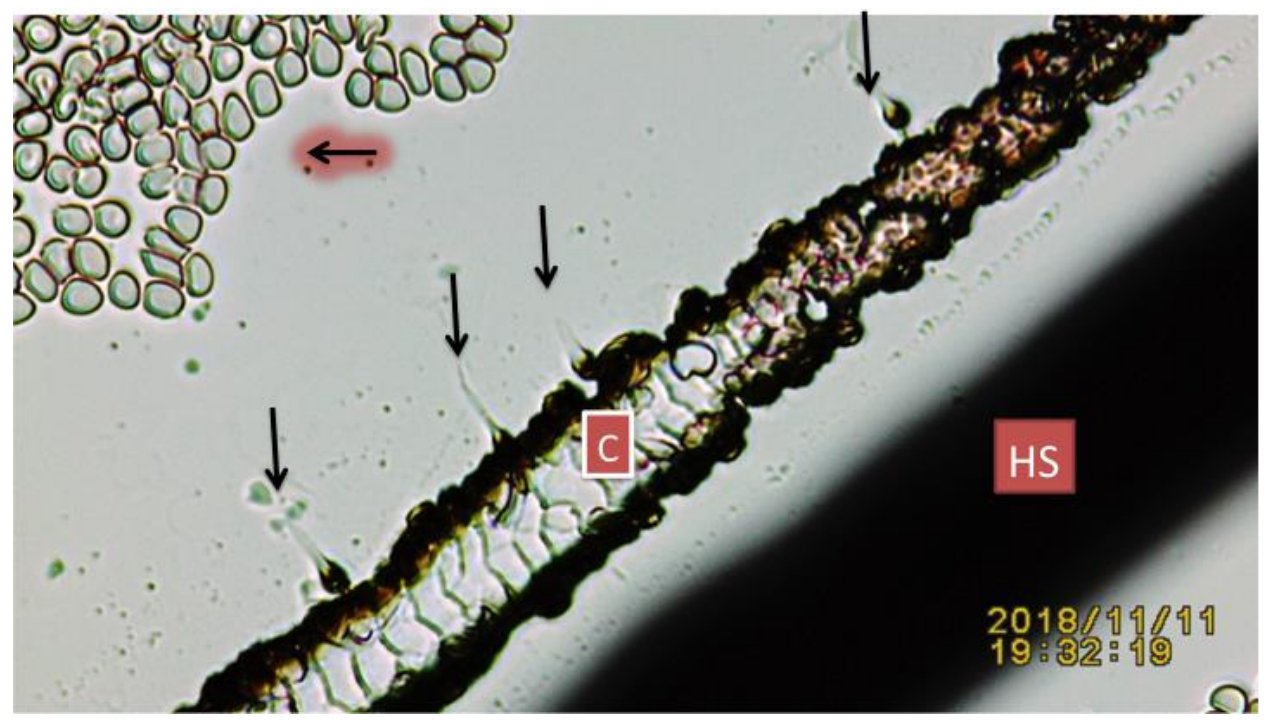

Figure S7: Image of female blood smear showing four teardrop RBCs attracted to hair shaft cuticles. Highlighted black arrow showing asymmetrical repulsion of RBCs. HS= Hair shaft proper detached drifting away from cuticles. Please compare with Figure 6 in text showing bilateral multiple teardrops in one of the male subjects.

\footnotetext{
*Corresponding author.

E-mail address: embi21@ att.net.
} 